\title{
Diversidad de flora vascular del Chocó Andino en el área de Selva Virgen, Ecuador
}

\section{(Diversity of vascular flora at Andino's Choco area in Selva Virgen)}

\author{
Ximena Aguirre Ulloa ${ }^{1}$, Alexandra Endara ${ }^{1}$
}

\begin{abstract}
Resumen:
El bosque junto a la Hostería Selva Virgen, ubicado en el noroccidente de Pichincha, zona del Chocó Andino, es uno de los sitios de alta biodiversidad (hotspot) del mundo; lo cual se determinó en el análisis de su flora vascular. Para dicho análisis se implementó un muestreo con transectos temporales de $50 \times 4$ y $50 \times 2$ m que cubren una superficie de 0.1 hectáreas. Se calcularon los índices de Sorensen, Simpson y Shannon, con el programa Past, obteniéndose los siguientes resultados: 159 especies (spp.) registradas, que corresponden a 121 géneros y 54 familias. Las familias con mayor número de especies fueron: Moraceae $15 \mathrm{spp}$, Rubiaceae $10 \mathrm{spp}$, Arecaceae $9 \mathrm{spp}$, Fabaceae $9 \mathrm{spp}$, Melastomataceae $8 \mathrm{spp}$ y Meliaceae $8 \mathrm{spp}$. La diversidad obtuvo un valor de 0.95 en el índice de Simpson, que se interpreta como alta diversidad. La riqueza fue de 0.33 , riqueza media. El índice de Sorensen identifica espacios que comparten el mayor número de especies. El endemismo es bajo: 6.25\%. La alta biodiversidad encontrada en el bosque, demuestra una riqueza biológica importante, que sugiere la realización de más estudios en esta zona poco explorada desde el punto de vista biológico.
\end{abstract}

Palabras clave: bosque; biodiversidad; flora vascular; riqueza biológica

\begin{abstract}
:
The forest near the Hostería Selva Virgen, located in Pichincha, northwestern Andean Chocó area, is one of the biodiversity hotspots in the world, reason that determined the analysis of the vascular flora in the zone. For this study we did a temporary transects of $50 \times 4$ and $50 \times 2 \mathrm{~m}$ covering an area of 0.1 hectares. We calculated Sorensen, Simpson and Shannon indexes, using the Past software, obtaining the following results: 159 species (spp.) registered corresponding to 121 genera and 54 families. Families with more species were: $15 \mathrm{spp}$ Moraceae, Rubiaceae 10 spp, Arecaceae 9 spp, Fabaceae 9 spp, Melastomataceae 8 spp and Meliaceae $8 \mathrm{spp}$. The diversity obtained has a value of 0.95 . It is interpreted in the Simpson index as a high diversity. Richness was 0.33 , interpreted as an average richness. Sorensen index identifies areas that share more species. Endemism is low: $6.25 \%$. The high biodiversity found in the studied forest shows an important biological richness; therefore more studies are suggested, because this zone has been rarely explored from the biological point of view.
\end{abstract}

Keywords: forest; biodiversity; vascular flora; biological richness

\footnotetext{
${ }^{1}$ Universidad Tecnológica Equinoccial, Quito - Ecuador ( \{xaguirre, maria.endara\} @ute.edu.ec )
} 


\section{Introducción}

La flora del Ecuador ha sido estudiada de manera sistemática desde el año de 1972 y, a partir de entonces, se han publicado 80 volúmenes de la colección Flora Ecuador Series (Revista publicada por la Universidad de Gotemburgo, de Suecia). En estas colecciones se ha encontrado entre 10$15 \%$ de nuevas especies para la ciencia botánica (Bravo, 2014). A pesar de la gran biodiversidad de flora del Ecuador, la tasa de deforestación de sus bosques es una de las más altas del mundo (Bravo, 2014); y, según Pitman et al. (2002), entre 19 a 46 especies de plantas se han extinguido en el Ecuador a lo largo de los últimos 250 años, debido principalmente a la pérdida de hábitat. Además, 282 especies (cerca del 7\% de la flora endémica del Ecuador) han sido calificadas como críticamente amenazadas, sobre todo aquellas ubicadas en los bosques costeros y en los Andes ecuatorianos (Pitman et al., 2002).

El bosque de Selva Virgen está localizado en el Piso Tropical Noroccidental, que corresponde a una de las regiones más húmedas del globo, el Chocó Andino, donde la precipitación anual alcanza, en algunos lugares, hasta 10000 mm (Cabrera y Willink, 1989, en Albuja et al., 2012). Es, además, uno de los sitios de alta diversidad biológica (Hot Spots) del mundo (Albuja et al., 2012). Estos bosques son parte de la región biogeográfica del Chocó, que se extiende desde Panamá, a lo largo del Pacífico colombiano, y se adentra en el noroccidente del Ecuador (García et al., 2014).

La extrema humedad del ambiente se debe a la acción de las corrientes marinas cálidas, como la corriente cálida El Niño (Fenómeno del Niño), cuya influencia se acentúa entre los meses de febrero hasta abril (Albuja et al., 2012). Este bosque es alto, denso y siempre verde, con el dosel frecuentemente de 30 m o más de altura y una alta diversidad de especies. La diversidad alfa de los árboles es menor que la encontrada en la Amazonía; sin embargo, la densidad y la diversidad de las epífitas son probablemente iguales o más altas en los bosques del noroeste del Ecuador (Neill 1995-2011, en Albuja et al., 2012).

El bosque del Chocó, de manera general, presenta tres estratos: alto, medio y bajo. El estrato superior o dosel está formado por árboles que superan los $30 \mathrm{~m}$ de altura. Entre los principales árboles del dosel están: chanul (Humiriastrum procerum), sande (Brosimum utile), peine de mono (Apeiba aspera), sangre de gallina (Otoba gracilipes), copal (Dacryodes occidentalis), figueroa (Carapa guianensis), y ceibo (Ceiba pentandra) (Albuja et al., 2012). El estrato medio está conformado principalmente por: gualte (Wettinia quinaria), chilalde (Luehea cymulosa), balsa (Ochroma pyramidale), y uva de monte (Pourouma bicolor chocoana) (Albuja et al., 2012).

El estrato inferior es heterogéneo y denso en herbáceas. Son frecuentes las familias Araceae, Melastomataceae y Cyclantaceae (Albuja et al., 2012). Algunas plantas trepadoras cubren los fustes de los árboles; entre ellas, destacan Philodendron verrucosum, $P$. rhodoaxis y Rhodospatha densinervia (Albuja et al., 2012). Conforme se asciende en las laderas, la vegetación disminuye 
progresivamente de tamaño. Es notable la abundancia de musgos, helechos, orquídeas, bromelias y otras epífitas (Albuja et al., 2012).

A pesar de la riqueza antes descrita, la región del Chocó enfrenta un dilema: por un lado, es considerada como uno de los "puntos calientes de biodiversidad del planeta"; por otro, se trata de los bosques más amenazados del Ecuador (García et al., 2014). Adicionalmente, en el noroccidente de la provincia de Pichincha, bajo la cota de los 1000 metros de altitud, no se han publicado estudios florísticos. Solo se ha encontrado la publicación de plantas útiles, realizada por Montserrat Ríos en 1993. Este estudio es uno de los pocos que se refiere a estos rangos altitudinales del noroccidente de Pichincha.

En este marco, surge la iniciativa de analizar la biodiversidad de flora vascular en el bosque adjunto a la Hostería Selva Virgen, ubicada en la zona noroccidental de la provincia de Pichincha, cercana a los bosques costeros del Ecuador, con la finalidad de conocer su importancia biológica.

\section{Metodología}

El presente trabajo emplea el método de transectos temporales de $50 \times 4$ y $50 \times 2 \mathrm{~m}$ que cubren una superficie de 0.1 hectárea. Los transectos se ubicaron en el bosque maduro (bosque siempreverde piemontano). Se realizaron muestreos generales con cuatro puntos de observación cuantitativos de $10 \mathrm{~m}^{2}$, que permitieran ampliar la visión de la riqueza botánica de la región con especies que no ingresasen en el muestreo cuantitativo. Se complementó el trabajo con colecciones generales y fotografías de varios especímenes.

\section{Fase de campo}

Esta fase se desarrolló de acuerdo con el esquema de inventarios de vegetación para el neotrópico, donde se realizan levantamientos de individuos iguales o mayores a $2.5 \mathrm{~cm}$ de DAP (Diámetro a la Altura del Pecho). El total de transectos sumaron 9, el primero de cinco transectos

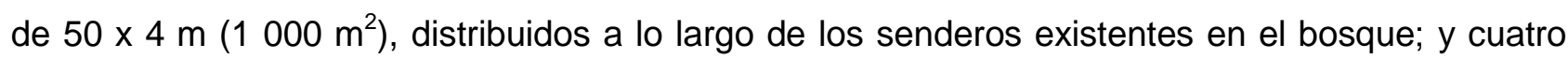
transectos de $50 \times 2 \mathrm{~m}\left(400 \mathrm{~m}^{2}\right)$, considerados como puntos de observación cuantitativa, para capturar especies que no se encontrasen en el muestreo de 0.1 hectáreas. En cada transecto se registró la altura estimada de los individuos y el diámetro del fuste. Se realizaron colecciones por especie, y de aquellos árboles que presentan dificultades para reconocerlos en el campo. Las muestras botánicas fueron prensadas y conservadas con alcohol al $75 \%$, para su posterior traslado al Herbario Nacional del Ecuador (QCNE), en la ciudad de Quito.

\section{Fase de gabinete y análisis de información}

Las muestras botánicas colectadas fueron transportadas al QCNE, donde fueron secadas e identificadas taxonómicamente, mediante comparación con especímenes que reposan en la colección de plantas vasculares y el uso de claves taxonómicas disponibles para algunos grupos y 
especies. La nomenclatura de las especies fue revisada en el Catálogo de Plantas Vasculares del Ecuador (Jørgensen \& León-Yánez, 1999), y la base electrónica de información botánica, w3 TROPICOS, del Missouri Botanical Garden (MO) (www.tropicos.org). El estatus de las especies endémicas se tomó del Libro Rojo de las Plantas Vasculares Endémicas del Ecuador (Valencia et al., 2000) y de información de la base electrónica TRÓPICOS.

\section{Área basal $\left(\mathrm{m}^{2}\right)$}

El área basal es la superficie de una sección transversal del tallo o tronco de un árbol a determinada altura del suelo; se expresa en $\mathrm{m}^{2}$ de material vegetal por unidad de superficie de terreno. En el estudio, se consideraron los individuos con diámetro mayor o igual a $2.5 \mathrm{~cm}$.

$$
\text { Área basal }=\frac{\pi * \mathrm{DAP}^{2}}{4}
$$

Donde:

DAP = Diámetro a la Altura del Pecho (convencionalmente $1.3 \mathrm{~m}$ desde el final de la raíz.)

$\Pi=$ constante 3.1416

\section{Densidad Relativa (DR \%)}

Se entiende como la proporción entre el número de individuos de una especie respecto del número total de individuos del muestreo.

$$
D R \%=\frac{\text { Número de individuos de una especie }}{\text { Número total de individuos en el muestreo }} \times 100
$$

\section{Dominancia Relativa (DMR \%)}

Se entiende como la proporción del área basal de una especie versus el área basal total de los individuos encontrados en el muestreo.

$$
D M R \%=\frac{\text { Área Basal de la especie }}{\text { Área Basal de todo el muestreo }} \times 100
$$

\section{Importancia Relativa (IMP)}

Es el resultado de la sumatoria de la Densidad Relativa (DR \%) y la Dominancia Relativa (DMR $\%$ ), dividido para 2. El valor máximo para el total de las especies es 100 . Se puede entonces considerar que especies con valores iguales o mayores a 10, caracterizan el muestreo, al tratarse de especies importantes y comunes del bosque. La alta biodiversidad del neotrópico y la baja dominancia de especies hacen que no sea común encontrar especies con valores de IMP igual a mayores a 10 . 


$$
I M R=\frac{D R \%+D M R \%}{2}
$$

Este índice se aplica en muestreos pequeños y es una forma simplificada del tradicional IVI (Índice de Valor de Importancia), que suma a los parámetros anteriores la Frecuencia Relativa (número de unidades de muestreo donde se registra la especie, dividido para el total de frecuencias de especies del muestreo).

\section{Riqueza y abundancia de especies}

El término riqueza se refiere a la abundancia de especies por individuo; es decir, el número de especies dividido por el número de individuos muestreados. Este dato permite realizar una comparación directa en cuanto a la diversidad (riqueza) de especies de individuos botánicos, aun cuando el número de individuos sea variable entre muestreos (el dato siempre es un valor entre 0 y 1; si todos los individuos de los muestreos fueran de especies diferentes, tendría un valor de 1; un valor de 0,5 significa una alta diversidad de especies).

$$
\text { Riqueza }=\frac{\text { Número de especies del sitio de muestreo }}{\text { Número de individuos del sitio de muestreo }}
$$

\section{Índice de similitud de Sorensen cualitativo}

Este índice permite realizar comparaciones en la composición de especies por su presencia/ausencia. Su valor va de 0 a 1 (0 a 100\%), donde el 1 (100\%) indica que todas las especies se comparten.

$$
I S S=\frac{2 C}{A+B}
$$

Dónde:

ISS = Índice de Similitud Sorensen

$\mathrm{A}=$ número de especies sitio 1

$\mathrm{B}=$ número de especies sitio 2

$\mathrm{C}=$ número de especies compartidas entre muestreo 1 y 2

La diversidad se analizó mediante el programa estadístico PAST v2.3, y se emplearon los índices de dominancia de Simpson, diversidad de Shannon y Equitatividad.

\section{Resultados y Discusión}

El muestreo total registró 159 especies (spp.) en 0,1 hectáreas, que corresponden a 121 géneros y 54 familias. Las familias con mayor número de especies fueron: Moraceae, con $15 \mathrm{spp}$. (moral, caucho, tillo y matapalo); Rubiaceae, con 10 spp. (café y cauje); Arecaceae, con 9 spp. (pambil, bísula y palmito); Fabaceae, con 9 spp. (familia de las leguminosas); Melastomataceae, con 8 spp. (colcas); y, Meliaceae, con 8 spp. (tangarés y cedro). 


\section{Caracterización florística por transecto}

\section{Transecto 1 (TR1)}

Bosque secundario maduro. Tiene la influencia de la plantación de palmito. La vegetación herbácea es densa con plantas de las familias Piperaceae, Rubiaceae y Araceae. Se encontró individuos de árboles juveniles y arbustos de Rubiaceae. Los árboles emergentes alcanzan los 30 $\mathrm{m}$ y los fustes diámetros de hasta $50 \mathrm{~cm}$. El dosel se estableció entre los 15 y $20 \mathrm{~m}$ de altura. Se registraron 23 especies correspondientes a 23 géneros y 16 familias. Las familias con mayor número de especies fueron: Arecaceae (3 especies), Malvaceae (2 spp.), Moraceae (2 spp.) y Urticaceae (2 spp.).

La especie que destacó es Wettinia quinaria, palma nativa que alcanza $25 \mathrm{~m}$ de alto y 20,4 cm de DAP, siendo la especie más frecuente en el transecto (Tabla 1).

Tabla 1. Especies que denotan mayor importancia relativa en TR1

\begin{tabular}{|l|c|c|c|c|c|}
\hline \multicolumn{1}{|c|}{ Especie } & $\begin{array}{c}\text { Máx de } \\
\text { altura }\end{array}$ & $\begin{array}{c}\text { Máx de } \\
\text { DAP }\end{array}$ & Frec. & $\begin{array}{c}\text { Área } \\
\text { basal }\end{array}$ & $\begin{array}{c}\text { Importancia } \\
\text { relativa }\end{array}$ \\
\hline $\begin{array}{l}\text { Wettinia quinaria (O.F. Cook \& } \\
\text { Doyle) Burret }\end{array}$ & 25 & 20,4 & 12 & 0,165 & 18,804 \\
\hline Theobroma gileri Cuatrec. & 17 & 27,7 & 5 & 0,180 & 12,098 \\
\hline Bactris gasipaes Kunth & 16 & 57,6 & 1 & 0,261 & 10,990 \\
\hline Sorocea trophoides W.C. Burger & 18 & 23,2 & 4 & 0,113 & 8,465 \\
\hline Pourouma bicolor Mart. & 20 & 35,7 & 2 & 0,137 & 7,304 \\
\hline Cyathea poeppigii (Hook.) Domin & 6 & 41,6 & 1 & 0,136 & 6,231 \\
\hline
\end{tabular}

\section{Transecto 2 (TR2)}

Bosque maduro, que se encuentra cerca de la segunda parada del sendero. La vegetación herbácea es densa, y presenta plantas de bijao (Maranthaceae), helechos, colcas (Melastomataceae) y aráceas. Los arbustos presentes corresponden a colcas (Melastomataceae) y cafetillos (Rubiaceae). El dosel es discontinuo y se encuentra entre los 22 y $20 \mathrm{~m}$. Los árboles emergentes superan los $30 \mathrm{~m}$ de alto y diámetros de hasta $70 \mathrm{~cm}$. Se registró un total de 30 especies, que corresponden a 27 géneros y 15 familias. Las familias con mayor número de especies son: Moraceae (7 spp.), Malvaceae (4 spp.), Urticaceae (3 spp.), Arecaceae (2 spp.), Myristicaceae (2 spp.) y Rubiaceae (2 spp.). La especie más importante es Iriartea deltoidea "pambil", palma nativa, considerada como la especie más frecuente (Tabla 2).

\section{Transecto 3 (TR3)}

Bosque maduro. Se ubica aproximadamente 100 metros al sur de la segunda parada del sendero. La vegetación herbácea es muy abundante, en espacios con claros de bosque. Las especies que sobresalen pertenecen a helechos, aráceas, cordoncillos (Piperaceae), heliconias (Heliconiaceae) y bijaos (Maranthaceae). Esta vegetación supera, en ciertas zonas, el $1.5 \mathrm{~m}$ de alto. El dosel es 
discontinuo, con un rango comprendido entre los 17 y $22 \mathrm{~m}$ de altura. Las especies emergentes superan los $30 \mathrm{~m}$ de alto, con individuos con diámetros de hasta $56 \mathrm{~cm}$.

Tabla 2. Especies que denotan mayor importancia relativa en TR2

\begin{tabular}{|l|c|c|c|c|c|}
\hline \multicolumn{1}{|c|}{ Especie } & $\begin{array}{c}\text { Máx de } \\
\text { altura }\end{array}$ & $\begin{array}{c}\text { Máx de } \\
\text { DAP }\end{array}$ & Frec. & $\begin{array}{c}\text { Area } \\
\text { basal }\end{array}$ & $\begin{array}{c}\text { Importancia } \\
\text { relativa }\end{array}$ \\
\hline Iriartea deltoidea Ruiz \& Pav. & 25 & 37,4 & 7 & 0,389 & 15,266 \\
\hline Neea parviflora Poepp. \& Endl. & 30 & 74,2 & 1 & 0,432 & 10,815 \\
\hline Castilla elastica Sessé ex Cerv. & 40 & 40 & 3 & 0,227 & 7,922 \\
\hline Inga coruscans Humb. \& Bonpl. ex Willd. & 30 & 55,6 & 2 & 0,261 & 7,807 \\
\hline Ficus obtusifolia Kunth & 15 & 40 & 2 & 0,175 & 5,821 \\
\hline $\begin{array}{l}\text { Pseudolmedia rigida (Klotzsch \& H. Karst.) } \\
\text { Cuatrec. }\end{array}$ & 25 & 19,7 & 5 & 0,053 & 5,756 \\
\hline
\end{tabular}

Se encontraron 27 especies, que pertenecen a 21 géneros y 13 familias. Las familias con mayor número de especies son: Areaceae (5 especies), Malvaceae (5 spp.), Fabaceae (3 spp.), Meliaceae (3 spp.) y Moraceae (2 spp.). La especie que destacó por el valor de importancia relativa fue Pourouma bicolor o "uva de monte". El árbol nativo alcanzó $40 \mathrm{~m}$ de alto, con $56.3 \mathrm{~cm}$ de DAP. Fue la segunda especie en frecuencia y la que mayor área basal presentó (Tabla 3).

Tabla 3. Especies que denotan mayor importancia relativa en TR3

\begin{tabular}{|l|c|c|c|c|c|}
\hline \multicolumn{1}{|c|}{ Especie } & $\begin{array}{c}\text { Máx de } \\
\text { Altura }\end{array}$ & $\begin{array}{c}\text { Máx de } \\
\text { DAP }\end{array}$ & Frec. & $\begin{array}{c}\text { Área } \\
\text { basal }\end{array}$ & $\begin{array}{c}\text { Importancia } \\
\text { relativa }\end{array}$ \\
\hline Pourouma bicolor Mart. & 40 & 56,3 & 3 & 0,259 & 14,963 \\
\hline $\begin{array}{l}\text { Carapa megistocarpa A.H. Gentry \& } \\
\text { Dodson }\end{array}$ & 20 & 41,3 & 2 & 0,136 & 8,409 \\
\hline Matisia coloradorum Benoist & 30 & 40,2 & 2 & 0,134 & 8,309 \\
\hline Welfia regia Mast. & 15 & 43,4 & 1 & 0,148 & 7,624 \\
\hline Sorocea trophoides W.C. Burger & 17 & 19,3 & 4 & 0,040 & 6,849 \\
\hline Oenocarpus bataua Mart. & 12 & 37,6 & 1 & 0,111 & 6,042 \\
\hline
\end{tabular}

\section{Transecto 4 (TR4)}

Bosque maduro. Localizado entre la segunda y tercera parada del sendero. La vegetación herbácea es densa, con individuos de aráceas, selaginelas, cordoncillos (Piperaceae) y rubiáceas. El dosel se ubica entre los 15 y $22 \mathrm{~m}$ de alto. Las especies emergentes superan los $30 \mathrm{~m}$ de alto. Los individuos presentaron diámetros relativamente pequeños, que alcanzan un valor máximo de $30 \mathrm{~cm}$; esto, debido a que el paisaje es dominado por la presencia de palmas (pambil, palma real, bísula), grupo que generalmente no presenta fustes muy gruesos.

En este transecto se registraron 28 especies, que pertenecen a 27 géneros y 16 familias; las familias con mayor número de especies fueron: Arecaceae (5 spp.), Moraceae (4 spp.), Malvaceae (3 spp.), Myristicaceae (2 spp.), Solanaceae (2 spp.) y Rubiaceae (2 spp.). La especie más importante fue Iriartea deltoidea "pambil", palma nativa, alcanzó los $40 \mathrm{~m}$ de alto y $30 \mathrm{~cm}$ de DAP; fue la segunda especie más frecuente y la primera con valor de área basal (Tabla 4). 
Tabla 4. Especies que denotan mayor importancia relativa en TR4

\begin{tabular}{|c|c|c|c|c|c|}
\hline Especie & $\begin{array}{l}\text { Máx de } \\
\text { Altura }\end{array}$ & $\begin{array}{l}\text { Máx de } \\
\text { DAP }\end{array}$ & Frec. & $\begin{array}{l}\text { Área } \\
\text { Basal }\end{array}$ & $\begin{array}{l}\text { Importancia } \\
\text { Relativa }\end{array}$ \\
\hline Iriartea deltoidea Ruiz \& Pav. & 40 & 30 & 6 & 0,312 & 18,029 \\
\hline $\begin{array}{l}\text { Brosimum utile subsp. occidentale } \\
\text { C.C. Berg }\end{array}$ & 20 & 32,4 & 5 & 0,156 & 10,877 \\
\hline Theobroma gileri Cuatrec. & 10 & 20,3 & 8 & 0,070 & 10,185 \\
\hline Welfia regia Mast. & 30 & 33,3 & 3 & 0,144 & 8,528 \\
\hline $\begin{array}{l}\text { Wettinia quinaria (O.F. Cook \& } \\
\text { Doyle) Burret }\end{array}$ & 15 & 11,9 & 5 & 0,037 & 6,122 \\
\hline $\begin{array}{lll}\begin{array}{l}\text { Astrocaryum } \\
\text { Bailey }\end{array} & \text { standleyanum } & \text { L.H. } \\
\end{array}$ & 10 & 40 & 1 & 0,126 & 5,943 \\
\hline
\end{tabular}

\section{Transecto 5 (TR5)}

Bosque secundario maduro. Se localiza aproximadamente a $100 \mathrm{~m}$ al suroeste de la tercera parada. La vegetación herbácea está conformada, principalmente, por aráceas y helechos, siendo menos densa que en otros sitios muestreados: alcanza los $50 \mathrm{~cm}$ de alto. El dosel es discontinuo y se establece entre los 20 y $22 \mathrm{~m}$ de alto. Los individuos emergentes son pocos, con $35 \mathrm{~m}$ de altura. El diámetro de los árboles obtuvo registros de hasta $80 \mathrm{~cm}$. La presencia de guarumos indica que existió un evento natural o antrópico en el pasado, que modificó el paisaje y las poblaciones vegetales en este sitio.

Se registraron 24 especies, que corresponden a 23 géneros y 12 familias. Las familias con mayor número de especies son: Moraceae (5 spp.), Arecaceae (4 spp.), Meliaceae (4 spp.) y Malvaceae (2 spp.). La especie con valor alto de acuerdo con el Índice de Importancia Relativa fue Brosimum utile subsp. occidentale "sande"; árbol nativo. Fue la tercera especie en frecuencia, acompañada de tres especies más; sin embargo, su área basal (biomasa) era mucho mayor, dado que los individuos presentan diámetros muy grandes en sus fustes (Tabla 5).

Tabla 5. Especies que denotan mayor importancia relativa en TR5

\begin{tabular}{|l|c|c|c|c|c|}
\hline \multicolumn{1}{|c|}{ Especie } & $\begin{array}{c}\text { Máx de } \\
\text { altura }\end{array}$ & $\begin{array}{c}\text { Máx de } \\
\text { DAP }\end{array}$ & Frec. & $\begin{array}{c}\text { Área } \\
\text { basal }\end{array}$ & $\begin{array}{c}\text { Importancia } \\
\text { relativa }\end{array}$ \\
\hline $\begin{array}{l}\text { Brosimum utile subsp. occidentale } \\
\text { C.C. Berg }\end{array}$ & 30 & 80,9 & 3 & 0,616 & 19,902 \\
\hline $\begin{array}{l}\text { Wettinia quinaria (O.F. Cook \& } \\
\text { Doyle) Burret }\end{array}$ & 20 & 28,8 & 9 & 0,244 & 16,033 \\
\hline Theobroma gileri Cuatrec. & 10 & 32 & 6 & 0,215 & 12,105 \\
\hline Iriartea deltoidea Ruiz \& Pav. & 20 & 39,9 & 3 & 0,144 & 7,037 \\
\hline Sp.2 & 36 & 50 & 1 & 0,196 & 6,393 \\
\hline Cecropia reticulata Cuatrec. & 20 & 25,3 & 3 & 0,062 & 4,810 \\
\hline
\end{tabular}

El conjunto de cinco transectos mostró a cuatro especies como las más importantes: Iriartea deltoidea, "pambil”, con el 10\% de acuerdo con el Índice de Importancia Relativa; seguido de Wettinia quinaria, "bísula", con 8.7\%; Brosimum utile subsp. occidentale, "sande", con 7.9\%; y, 
Theobroma gileri, "cacao de monte", con 7.6\%. Estas fueron las especies más comunes y de mayor diámetro de fuste.

\section{Transecto 6 (TR6)}

Bosque maduro con pendiente, continuando por el sendero hacia la plantación de cacao, $100 \mathrm{~m}$ aproximadamente desde el transecto 5. La vegetación herbácea es densa con plantas de arácea, ciclantácea, piperácea (cordoncillos) y marantácea (bijaos). El dosel era discontinuo, con árboles de hasta $20 \mathrm{~m}$ de alto. El sotobosque se encontraba entre los 8 y $15 \mathrm{~m}$ de altura. El diámetro de los árboles alcanza los $30 \mathrm{~cm}$. Se registraron 22 especies, que corresponden a 21 géneros y 12 familias. Las familias con mayor número de especies fueron: Moraceae (3 spp.), Myristicaceae (3 spp.), Rubiaceae (3 spp.), Arecaceae (2 spp.), Malvaceae (2 spp.), Meliaceae (2 spp.) y Urticaceae (2 spp.).

La especie con mayor importancia relativa fue Cecropia reticulata "guarumo"; árbol nativo, con un solo registro, radicando su importancia en el valor del área basal al ser el individuo con mayor diámetro en el transecto (Tabla 6).

Tabla 6. Especies que denotan mayor importancia relativa en TR6

\begin{tabular}{|l|c|c|c|c|c|}
\hline \multicolumn{1}{|c|}{ Especie } & $\begin{array}{c}\text { Máx de } \\
\text { Altura }\end{array}$ & $\begin{array}{c}\text { Máx de } \\
\text { DAP }\end{array}$ & Frec. & $\begin{array}{c}\text { Área } \\
\text { Basal }\end{array}$ & $\begin{array}{c}\text { Importancia } \\
\text { Relativa }\end{array}$ \\
\hline Cecropia reticulata Cuatrec. & 25 & 34,4 & 1 & 0,093 & 12,258 \\
\hline $\begin{array}{l}\text { Brosimum utile subsp. occidentale } \\
\text { C.C. Berg }\end{array}$ & 8 & 8,3 & 6 & 0,019 & 10,799 \\
\hline Virola reidii Little & 10 & 30,1 & 1 & 0,071 & 9,720 \\
\hline $\begin{array}{l}\text { Wettinia quinaria (O.F. Cook \& } \\
\text { Doyle) Burret }\end{array}$ & 15 & 13,5 & 3 & 0,035 & 8,379 \\
\hline Perebea xanthochyma H. Karst. & 13 & 12,8 & 3 & 0,029 & 7,614 \\
\hline Welfia regia Mast. & 20 & 25,5 & 1 & 0,051 & 7,379 \\
\hline
\end{tabular}

\section{Transecto 7 (TR7)}

Bosque maduro, con influencia de claro de bosque. Se encuentra en el sendero que conduce a la plantación de cacao. La vegetación herbácea es poco densa con plantas de rubiáceas, aráceas, ciclantáceas, marantáceas (bijaos) y helechos. El dosel es muy abierto, entre los 20 y $22 \mathrm{~m}$ de altura. Los árboles emergentes miden $40 \mathrm{~m}$. Los diámetros máximos de los fustes se encuentran entre los 35 y $150 \mathrm{~cm}$.

Se registraron 19 especies, que pertenecen a 18 géneros y 12 familias. Las familias con mayor número de especies fueron: Urticaceae (3 spp.), Arecaceae (2 spp.), Malvaceae (2 spp.), Melastomataceae (2 spp.), Moraceae (2 spp.) y Sapotaceae (2 spp.). La especie sobresaliente fue Coussapoa cf. duquei, "mata palo", estranguladora nativa. Con solo un individuo obtuvo el valor más grande de área basal en el transecto (Tabla 7). 
Tabla 7. Especies que denotan mayor importancia relativa en TR7

\begin{tabular}{|l|c|c|c|c|c|}
\hline \multicolumn{1}{|c|}{ Especie } & $\begin{array}{c}\text { Máx de } \\
\text { altura }\end{array}$ & $\begin{array}{c}\text { Máx de } \\
\text { DAP }\end{array}$ & Frec. & $\begin{array}{c}\text { Área } \\
\text { basal }\end{array}$ & $\begin{array}{c}\text { Importancia } \\
\text { relativa }\end{array}$ \\
\hline Coussapoa cf. duquei Standl. & 40 & 150 & 1 & 1,767 & 40,308 \\
\hline Pourouma bicolor Mart. & 40 & 36 & 2 & 0,134 & 6,630 \\
\hline Trema micrantha (L.) Blume & 20 & 25,6 & 2 & 0,067 & 5,159 \\
\hline Iriartea deltoidea Ruiz \& Pav. & 25 & 24 & 2 & 0,057 & 4,934 \\
\hline Meriania cf. maxima Markgr. & 15 & 20,5 & 2 & 0,051 & 4,822 \\
\hline $\begin{array}{l}\text { Pleuranthodendron lindenii (Turcz.) } \\
\text { Sleumer }\end{array}$ & 15 & 12 & 2 & 0,016 & 4,059 \\
\hline
\end{tabular}

\section{Transecto 8 (TR8)}

Bosque maduro, aproximadamente a 130 m de la plantación de cacao. La vegetación herbácea no es muy densa, con individuos de ciclantácea, arácea y helechos. El dosel es muy abierto, con pocos individuos que alcanzan los $20 \mathrm{~m}$ de alto. Los árboles emergentes superan los $30 \mathrm{~m}$ de alto. Se encontraron 19 especies pertenecientes a 19 géneros y 13 familias. Las familias con mayor número de especies fueron: Moraceae (4 spp.), Arecaceae (3 spp.) y Lecythidaceae (2 spp.). La especie que destacó fue Inga inicuil, "guaba"; árbol nativo. Fue la quinta especie más frecuente, pero con diámetros grandes de fuste (Tabla 8).

Tabla 8. Especies que denotan mayor importancia relativa en TR8

\begin{tabular}{|l|c|c|c|c|c|}
\hline \multicolumn{1}{|c|}{ Especie } & $\begin{array}{c}\text { Máx de } \\
\text { altura }\end{array}$ & $\begin{array}{c}\text { Máx de } \\
\text { DAP }\end{array}$ & Frec. & $\begin{array}{c}\text { Área } \\
\text { basal }\end{array}$ & $\begin{array}{c}\text { Importancia } \\
\text { relativa }\end{array}$ \\
\hline $\begin{array}{l}\text { Inga inicuil Schltdl. \& Cham. ex G. } \\
\text { Don }\end{array}$ & 40 & 62,2 & 2 & 0,357 & 28,261 \\
\hline Iriartea deltoidea Ruiz \& Pav. & 15 & 35,6 & 2 & 0,104 & 10,216 \\
\hline Grias peruviana Miers & 5 & 10,4 & 5 & 0,020 & 8,401 \\
\hline Virola reidii Little & 20 & 30,7 & 2 & 0,076 & 8,220 \\
\hline Cecropia reticulata Cuatrec. & 8 & 9,9 & 4 & 0,019 & 6,895 \\
\hline $\begin{array}{l}\text { Wettinia quinaria (O.F. Cook \& } \\
\text { Doyle) Burret }\end{array}$ & 12 & 16,3 & 3 & 0,037 & 6,806 \\
\hline
\end{tabular}

\section{Transecto 9 (TR9)}

Bosque maduro. Se localiza a $80 \mathrm{~m}$ aproximadamente de la plantación de palmito. La vegetación herbácea no es muy densa, con dominio de aráceas en el suelo, así como epífitas en los fustes de los árboles. El dosel se encuentra entre los 15 y 20 m de altura; los individuos emergentes medían $30 \mathrm{~m}$. Se registraron 21 especies que corresponden a 20 géneros y 13 familias. Las familias con mayor número de especies fueron: Moraceae (6 spp.), Arecaceae (3 spp.) y Urticaceae (2 spp.). La especie con mayor importancia relativa fue Meriania cf. maxima, árbol nativo. Fue la sexta especie más frecuente con DAP muy grande (Tabla 9). 
Tabla 9. Especies que denotan mayor importancia relativa en TR9

\begin{tabular}{|l|c|c|c|c|c|}
\hline \multicolumn{1}{|c|}{ Especie } & $\begin{array}{c}\text { Máx de } \\
\text { altura }\end{array}$ & $\begin{array}{c}\text { Máx de } \\
\text { DAP }\end{array}$ & Frec. & $\begin{array}{c}\text { Área } \\
\text { basal }\end{array}$ & $\begin{array}{c}\text { Importancia } \\
\text { relativa }\end{array}$ \\
\hline Meriania cf. maxima Markgr. & 30 & 40 & 2 & 0,155 & 16,302 \\
\hline Welfia regia Mast. & 12 & 12 & 5 & 0,043 & 10,682 \\
\hline Cecropia reticulata Cuatrec. & 18 & 19,4 & 3 & 0,057 & 9,189 \\
\hline $\begin{array}{l}\text { Wettinia quinaria (O.F. Cook \& } \\
\text { Doyle) Burret }\end{array}$ & 20 & 12 & 4 & 0,037 & 8,750 \\
\hline Iriartea deltoidea Ruiz \& Pav. & 25 & 24 & 2 & 0,053 & 7,422 \\
\hline Castilla elastica Sessé ex Cerv. & 20 & 28,1 & 1 & 0,062 & 6,813 \\
\hline
\end{tabular}

\section{Abundancia y riqueza}

En 0.1 de hectárea de bosque siempreverde piemontano, adjunto a la Hostería Selva Virgen, las cuatro especies más comunes fueron: Wettinia quinaria o "bísula", Theobroma gileri o "cacao de monte", Iriartea deltoidea o "pambil", y Brosimum utile subsp. occidentale o "sande", con individuos de diámetros mayores o iguales a $2.5 \mathrm{~cm}$ (Figura 1).

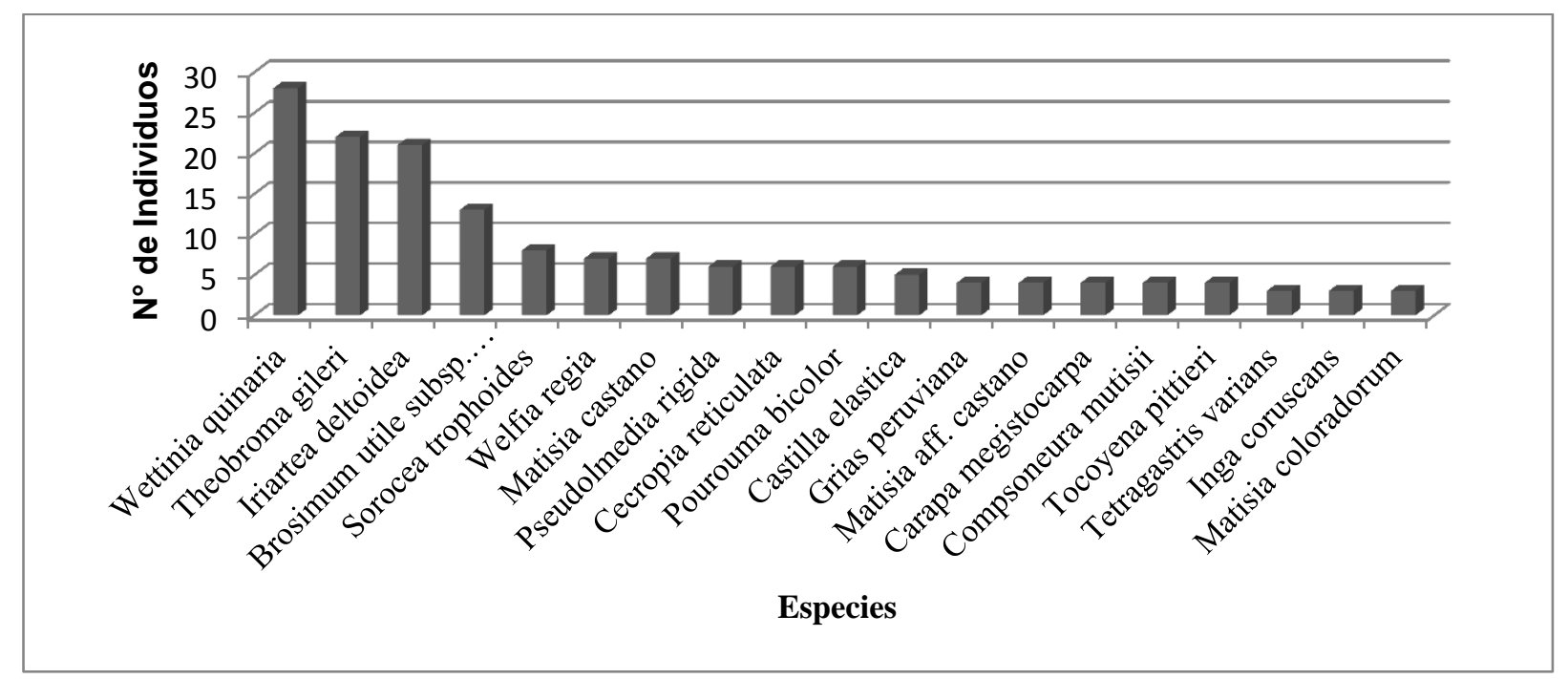

Figura 1. Listado de 20 especies más abundantes en el 0.1 de hectárea de bosque siempreverde piemontano adjunto a la Hostería Selva Virgen

La escasa publicación de estudios en la cota de los $300 \mathrm{~m}$ de altitud dificulta comparar la composición florística entre localidades. Estudios con transectos de 0.1 hectáreas en bosque siempreverde piemontano, en Esmeraldas, en el rango de 140-170 m de altitud, registraron entre 43 y 66 especies, un número de individuos que varía entre 170 a 264, en el sector Loma El Botadero. El bosque mostraba signos de explotación forestal. La especie más abundante es Wettinia quinaria o "bísula" (Cerón 2001).

En la localidad cooperativa Rumiñahui, provincia de Imbabura, a $550 \mathrm{~m}$ de altitud se registraron 56 especies con 126 individuos; las más comunes fueron Carapa guianensis o "tangaré" e Iriartea deltoidea o "pambil" (Cerón \& Yánez 2001). Esta última especie fue la tercera más frecuente en el 
bosque. En el río Sardinas, provincia de Pichincha a 770 m, se registraron 61 especies con 208 individuos; las más frecuentes fueron Iriartea deltoidea o "pambil" y Theobroma gileri o "cacao de monte" (Cerón \& Ojeda 2006), la tercera y segunda especies, respectivamente, más frecuentes en el presente estudio.

\section{Diversidad}

La diversidad obtuvo un valor de 0.95 en el Índice de Dominancia de Simpson, que se interpreta como diversidad alta (Tabla 10). El estudio realizado en Loma El Botadero empleó el Índice Recíproco de Simpson para el análisis, y se obtuvieron valores de diversidad entre media a muy diversa para la localidad de El Botadero, diversidad media para la cooperativa Rumiñahui y diversidad media para la zona baja del río Sardinas.

El índice empleado y las diferentes altitudes de las otras localidades no permiten comparar estos valores y obtener conclusiones contundentes. A pesar de ello se puede indicar que el bosque se encuentra en buen estado de conservación y tiene alta diversidad florística. La equitatividad muestra que la distribución de las especies en el espacio de muestreo alcanza el 86\%. La riqueza obtuvo un valor de 0.33 que se interpreta como riqueza media.

Tabla 10. Índices de diversidad y riqueza programa PAST v2.3

\begin{tabular}{|l|c|}
\hline \multicolumn{2}{|c|}{ Décima de Hectárea } \\
\hline Taxa_S & 82 \\
\hline Individuals & 244 \\
\hline Dominance_D & 0,04058 \\
\hline Shannon_H & 3,803 \\
\hline Simpson_1-D & 0,9594 \\
\hline Equitability_J & 0,8631 \\
\hline Riqueza (calculada con fórmula) & 0,336 \\
\hline
\end{tabular}

El Índice de Similitud de Sorensen permite identificar espacios que comparten mayor número de especies. En el muestreo ningún transecto compartía el $50 \%$ de las especies existentes en 0.1 hectárea. Los transectos que compartían más especies fueron TR2-TR4 con el 37.9\%, seguidos de TR3-TR4 con 36.3\% (Tabla 11).

Tabla 11. Índice de Similitud de Sorensen para 0.1 hectárea

\begin{tabular}{|l|c|c|c|c|c|}
\hline & TR1 & TR2 & TR3 & TR4 & TR5 \\
\hline TR1 & 1 & & & & \\
\hline TR2 & 0,2264 & 1 & & & \\
\hline TR3 & 0,32 & 0,2807 & 1 & & \\
\hline TR4 & 0,2745 & 0,3793 & 0,3636 & 1 & \\
\hline TR5 & 0,2553 & 0,2963 & 0,2745 & 0,3462 & 1 \\
\hline
\end{tabular}


La Figura 2, de agrupación por transecto, muestra que TR3 difiere en cuanto a la composición de especies, versus los otros transectos. En este punto de muestreo no existía mucho dominio de las palmas; lo más similares fueron TR4-TR5 (Figura 2).

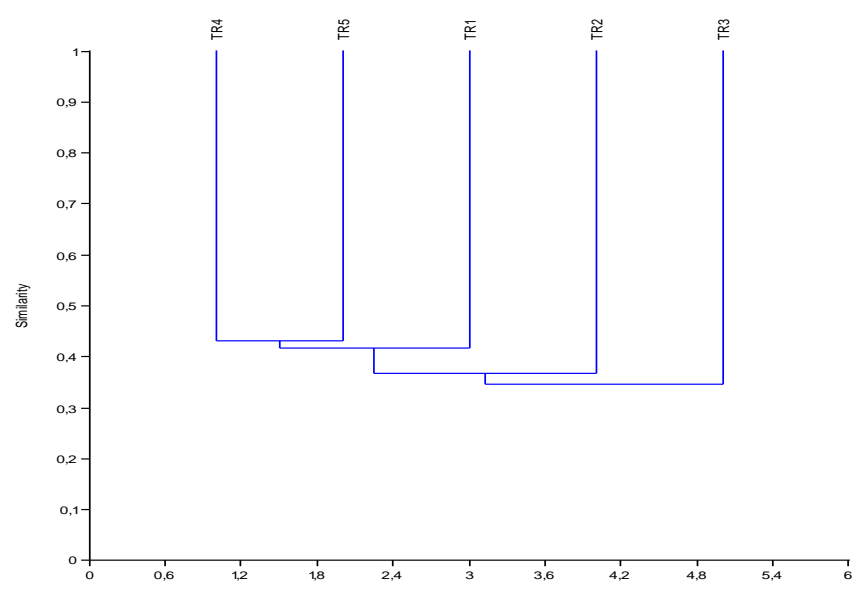

Figura 2. Agrupamiento de transectos por lincaje simple y medida de Bray-Curtis

Los cuatros transectos de $50 \times 2 \mathrm{~m}$ que se emplearon para reconocer otros ambientes muestran poca similitud en la composición de especies. Los más importantes fueron TR6-TR8 con el 39\%; y, TR6-TR9 con el $37.2 \%$ (Tabla 12).

Tabla 12. Índice de Similitud de Sorensen para análisis de punto de observación

\begin{tabular}{|l|c|c|c|c|}
\hline & TR6 & TR7 & TR8 & TR9 \\
\hline TR6 & 1 & & & \\
\hline TR7 & 0,3415 & 1 & & \\
\hline TR8 & 0,3902 & 0,3684 & 1 & \\
\hline TR9 & 0,3721 & 0,3 & 0,45 & 1 \\
\hline
\end{tabular}

La Figura 3, de agrupación por transecto, muestra que TR7 difiere en cuanto a la composición de especies, versus los otros transectos. Los más similares fueron TR8-TR9.

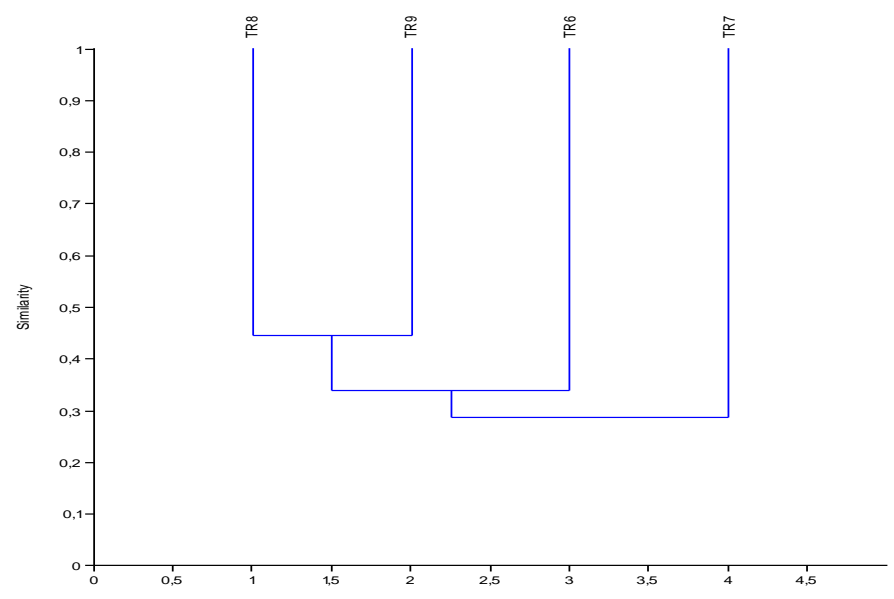

Figura 3. Agrupamiento de transectos por lincaje simple y medida de Bray-Curtis 


\section{Endemismo}

Se registró un total de 9 especies endémicas (Tabla 13) dentro de las categorías de amenaza de la UICN. Una especie, la Erythrina aff. smithiana, no pudo ser confirmada al no encontrarse fértil, y tiene categoría de Preocupación Menor (LC). Estas especies presentan pocos individuos: existen, como máximo, cuatro individuos en el espacio total de muestreo. Dos especies son vulnerables (VU), cuatro se encuentran en peligro (EN) y dos están casi amenazadas (NT).

El número de especies endémicas es bajo, pero se debe tomar en cuenta que el muestreo se centró en especies fértiles, arbustos y árboles. Se discriminó el grupo de las herbáceas y epífitas, donde se concentra el mayor número de especies endémicas en el Ecuador.

Tabla 13. Listado de especies endémicas y categoría de amenaza UICN

\begin{tabular}{|l|l|l|}
\hline \multicolumn{1}{|c|}{ Familia } & \multicolumn{1}{c|}{ Especie } & \multicolumn{1}{c|}{ Estatus } \\
\hline Annonaceae & Klarobelia megalocarpa Chatrou & EN A4c \\
\hline Araceae & Anthurium falcatum Sodiro & NT \\
\hline Fabaceae & Inga silanchensis T.D. Penn. & VU B1ab(iii) \\
\hline Lecythidaceae & Gustavia dodsonii S.A. Mori & EN A4c \\
\hline Bombacaceae & Matisia coloradorum Benoist & EN A4c \\
\hline Bombacaceae & Matisia grandifolia Little & EN A4c \\
\hline Melastomataceae & Miconia explicita Wurdack & VU A4c; B1ab(iii) \\
\hline Meliaceae & Carapa megistocarpa A.H. Gentry \& Dodson & EN A4c \\
\hline Olacaceae & Heisteria asplundii Sleumer & NT \\
\hline
\end{tabular}

\section{Conclusiones y Recomendaciones}

Las familias más importantes presentes en el bosque de Selva Virgen por el número de especies son: Moraceae, Rubiaceae, Arecaceae, Fabaceae, Melastomataceae y Meliaceae. Son evidentes árboles emergentes de hasta $40 \mathrm{~m}$. El dosel tiene una altura de entre 20 a $25 \mathrm{~m}$, los fustes se encuentran cubiertos por plantas epífitas de Cyclanthaceae, Araceae y helechos, principalmente. Las especies comunes en el bosque son: Wettinia quinaria o "bisula", Theobroma gileri o "cacao de monte", Iriartea deltoidea o "pambil", Brosimum utile subsp. occidentale o "sande", Sorocea trophoides y Welfia regia o "palma real".

La riqueza y diversidad del bosque es alta, con dominio de cuatro especies de un total de 82 registradas para el muestreo de 0.1 hectáreas, y 159 especies total entre colecciones generales y transectos. El endemismo es bajo, equivalente a $6.25 \%$ del total de especies. Debido a que se encontró una diversidad alta en el estudio desarrollado se puede determinar que Selva Virgen tiene importancia biológica relevante, lo que sugiere la realización de más estudios en los últimos fragmentos de bosque que se encuentran en el sector. 


\section{Bibliografía}

Albuja, L., A. Almendáriz, R. Barriga, L. D. Montalvo, F. Cáceres y J. L. Román. (2012): Fauna de Vertebrados del Ecuador. Quito, Instituto de Ciencias Biológicas. Escuela Politécnica Nacional.

Bravo, E. (2014): La biodiversidad en el Ecuador. Quito, Editorial ABYA YALA, Universidad Politécnica Salesiana.

Cerón, C. E. 2001. Caracterización botánica de la comunidad Playa de oro, cuenca del río Santiago, provincia de Esmeraldas. Cinchonia 2(1): 30-65.

Cerón, C. E. \& I. B. Ojeda. 2006. Diversidad florística de un bosque nuboso en Pacto, PichinchaEcuador. Cinchonia 7(1): 16-27.

Cerón, C.E. \& M. Yánez. 2001. Diversidad y especies frecuentes en los remanentes de la parte baja de la cordillera de Toisan, implicaciones para su conservación y manejo. Cinchonia (Quito) 2(1): 66-82.

Pitman, N., P. Jorgensen, R. Williams, S. León, R. Valencia (2002): Extinction-Rate Estimates for a Modern Neotropical Flora. Conservation Biology 16 (5), 1427-1431.

Ríos, M. (1993): Plantas útiles en el Noroccidente de Pichincha Etnobotánica del Caserío Álvaro Pérez Intriago y la Reserva Forestal ENDESA. Hombre y Ambiente 26 (VII), 7-184.

Valencia, R. N. Pitman, S. León-Yánez \& P.M. Jorgensen. 2000. Libro Rojo de las Plantas Endémicas del Ecuador 2000. Publications of QCA Herbarium, Pontificia Universidad Católica del Ecuador, Quito. 